\title{
A Novel Method of Evaluating the Frequency Response of a Photoacoustic Cell
}

\author{
Mariusz Suchenek
}

Received: 18 November 2013 / Accepted: 23 April 2014 / Published online: 25 May 2014

C The Author(s) 2014. This article is published with open access at Springerlink.com

\begin{abstract}
The frequency response of a photoacoustic cell can be evaluated by measuring the output amplitudes point-by-point at different modulation frequencies, by measuring the pulse response, or by recording the cell response to a chirp stimulation, in which the light modulation signal sweeps within a given range of frequencies. This paper presents yet another method, in which the stimulation signal consists of a number of sine waves with different frequencies. A theoretical description is presented, and properties of the techniques are discussed and compared with the experimental results.
\end{abstract}

Keywords Frequency response - Photoacoustic measurement

\section{Introduction}

During photoacoustic measurements, a measured specimen is usually placed in an acoustically isolated cell in order to increase signal and suppress noise [1,2]. The cell often operates as an acoustic resonator [3], and its properties significantly affect the measurement results. This paper describes the process of characterization of a resonant acoustic cell, in particular, determination of the frequency response of the cell [4].

When measuring the performance of an acoustic resonator, it is important to measure its frequency response in a specified frequency range with the accuracy as high as possible. On the basis of such a measurement, the parameters of the photoacoustic cell, such as the Q-factor, resonance frequency, or attenuation of the external noise,

M. Suchenek $(\varangle)$

Institute of Electronic Systems, Warsaw University of Technology,

Nowowiejska 15/19, 00-665Warsaw, Poland

e-mail: m.suchenek@ise.pw.edu.pl 
are determined. Those parameters should be also controlled during the measurement process in order to maintain an optimal operating point [5].

The gas concentration and temperature [6,7] affect the speed of sound which influences the resonant frequency of the photoacoustic cell. Therefore, the excitation frequency of the modulated light beam should be maintained in the vicinity of the resonance frequency of the photoacoustic cell [8].

The frequency response of the cell can be determined by different methods such as scanning point-by-point, measuring of a pulse response $[9,10]$, or by the chirp stimulation [11]. Each of the mentioned approaches has advantages and disadvantages. In the point-by-point method, the amplitude of the PA signal is evaluated independently at each frequency in separate measurements. In order to accurately determine the cell response, the measurements should usually cover a wide frequency range. As a result, such a method is time consuming, especially taking into consideration the need to average measurements. In the pulse method, a measured amplitude and phase response depend on a length of the pulse [10]. Stimulation of the resonant photoacoustic cell with such a signal results in deformation of the obtained characteristic, imposing the requirement of an additional correction. Further, in addition, the signal-to-noise ratio of the excitation signal is not constant over the entire frequency range.

\section{Description of Proposed Method}

This paper presents another approach to evaluate the frequency response of a photoacoustic cell. For this purpose, a complex excitation signal, combining multiple sine waves of different frequencies, is used. By inverse Fourier analysis, any signal can be decomposed and described by a combination of sine waves. By inverse Fourier analysis, it is possible to synthesize a signal with a required specific spectrum. From the point of view of the frequency response, determination of this spectrum could be limited only to the required frequency range. The amplitude and phase of the excitation signal could be programmed depending on the requirements. The excitation signal described in the time domain consists of a bunch of sinusoidal signals summed according to the equation,

$$
\begin{aligned}
x(t) & =A_{1} \sin \left(2 \pi f_{1} t+\varphi_{1}\right)+A_{2} \sin \left(2 \pi f_{2} t+\varphi_{2}\right)+\cdots+A_{N} \sin \left(2 \pi f_{N} t+\varphi_{N}\right) \\
& =\sum_{n=1}^{N} A_{n} \sin \left(2 \pi f_{n} t+\varphi_{n}\right),
\end{aligned}
$$

where $n$ is the frequency index, $f_{n}$ is the value of the frequency for index $n, A_{n}$ is the amplitude of the sine wave with index $n, \varphi_{n}$ is the phase of the sine wave with index $n$, and $t$ is the time.

In the presented method, the induced optical signal should be amplitude modulated. The modulation signal should be periodic. For the specific case of excitation with a single frequency $(N=1)$, the optical signal consists of a single sine wave at a fixed frequency. Such a signal corresponds to the point-by-point measurement method. In the case of a measurement of the frequency response within the range from $500 \mathrm{~Hz}$ 

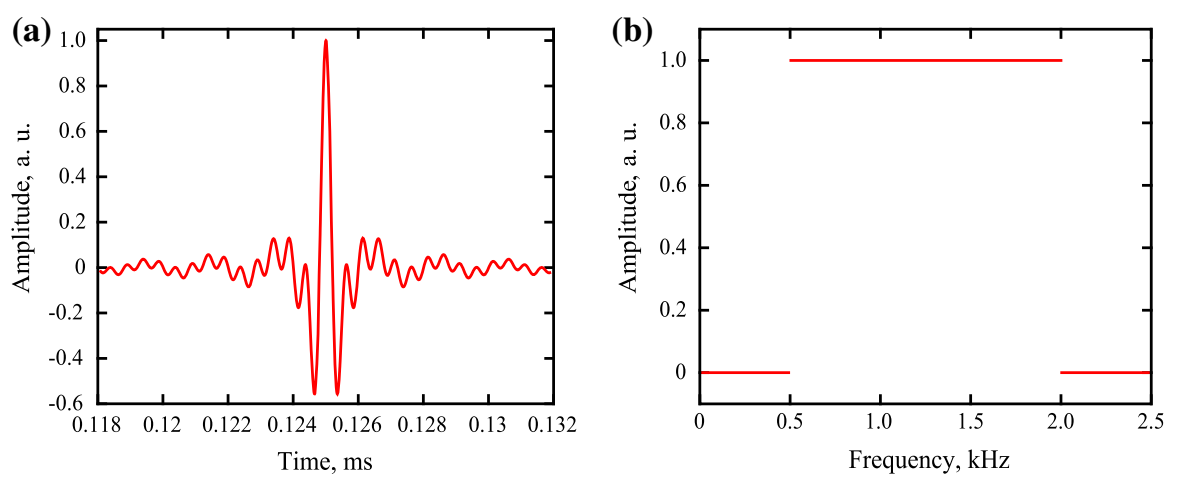

Fig. 1 Excitation signal in (a) time domain and (b) frequency domain

to $2 \mathrm{kHz}$ with $4 \mathrm{~Hz}$ resolution, the point-by-point method required 376 independent measurements. In contrary to the other methods, in the present method, the frequency response can be obtained in a single measurement-from a single excitation. The excitation signal is composed of the 376 sine waves, each with the same amplitude. Figure 1 shows a shape of the excitation signal amplitude and its spectrum.

\section{Experimental Verification}

In order to verify the proposed method, the frequency response of the same photoacoustic cell was measured using both: the novel approach as well as the point-by-point method. The experimental setup with a photoacoustic Helmholtz resonator is presented in Figs. 2 and 3.

In the experimental setup, a LED diode was used, in which change of the current allows simple modulation of the optical power. The frequency response of the resonator measured by the two methods is illustrated in Fig. 5. In the case of the method with superposition of sine waves, the modulation waveform was synthesized according to

Fig. 2 Sketch of a photoacoustic resonator used in the measurements

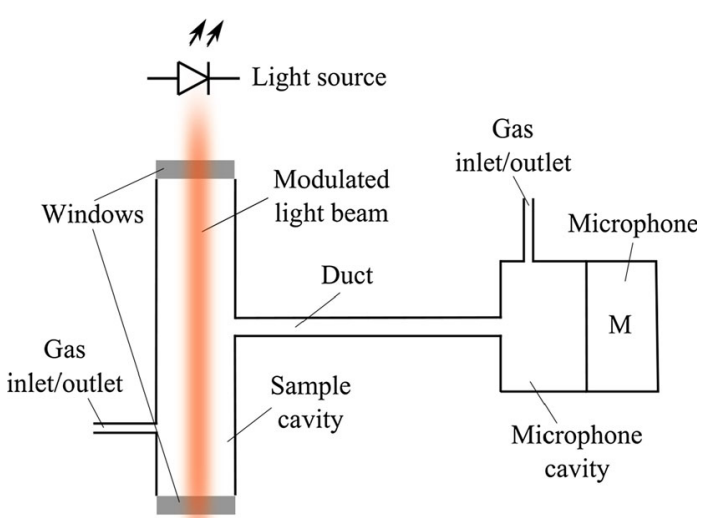


Fig. 3 Photograph of the tested photoacoustic resonator

Fig. 4 Photoacoustic response obtained from the measured cell stimulated by the complex signal
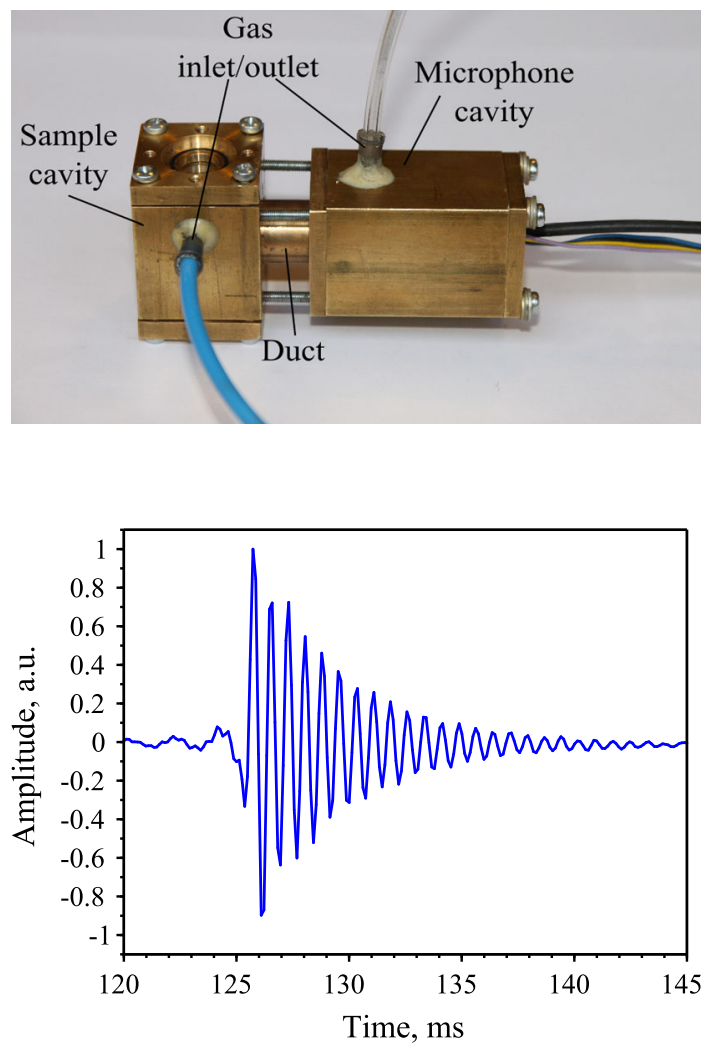

Eq. 1, converted to current, and added to a bias current driving the LED diode. The signal was synthesized in such a way that the frequency range of $0.5 \mathrm{kHz}$ to $2.0 \mathrm{kHz}$ was covered. The period of the modulation signal was $250 \mathrm{~ms}$. The photoacoustic signal from the microphone was averaged 100 times; thus, the whole measurement took $25 \mathrm{~s}$. The signal obtained in the time domain is presented in Fig. 4. After recording the photoacoustic signal, the Fourier transformation was applied to the collected data, resulting in the first harmonic at $4 \mathrm{~Hz}$, the second at $8 \mathrm{~Hz}$, the third at $12 \mathrm{~Hz}$, etc. (resolution of $4 \mathrm{~Hz}$ ).

In the case of the point-by-point method, the measurements started at $300 \mathrm{~Hz}$ and ended at $2.6 \mathrm{kHz}$ with a step of about $4 \mathrm{~Hz}$, and each measurement was averaged 100 times. From each measurement, eight samples per period were taken, from which the amplitude was calculated. The whole measurement took about 8 min including signal acquisition time. The frequency response displayed in the Fig. 5 was limited to the range of interest, i.e., $0.3 \mathrm{kHz}$ to $2.6 \mathrm{kHz}$.

It can be easily seen that results of both methods are practically identical within a specified frequency range. Outside the considered frequency range, the characteristic differs. The method with a complex excitation signal starts at $500 \mathrm{~Hz}$ and ends at 2 $\mathrm{kHz}$, confirming correct synthesis of the stimulation signal. 
Fig. 5 Comparison of the frequency responses obtained experimentally with the point-by-point method and superposition of sine wave

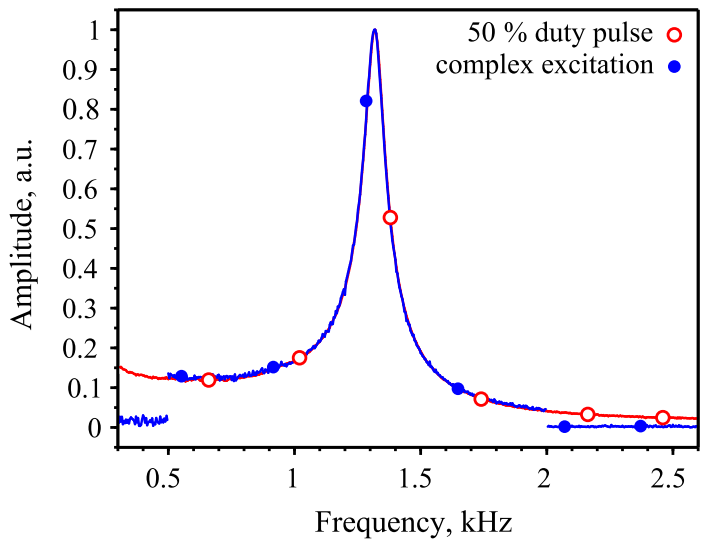

\section{Conclusions}

The presented method uses a complex excitation signal, and allows the frequency response of a photoacoustic cell to be determined in a single measurement. The optical power of the excitation signal can be modulated in such a way that the optical signal is a composition of a sine wave with different frequencies and equal amplitudes. The presented method gives the same results as the traditional point-by-point measurements (Fig. 5). The advantage of the presented method is that the spectrum of the excited signal is limited to a required frequency range. All frequencies included in the frequency response are generated at the same time, which provides the same measuring conditions for all the frequencies. In comparison to the point-by-point method, the measurement time in the presented approach is much shorter and is comparable to the time required by the pulse method (yet, the approach is free from the inconvenience induced by the pulse measurements related to pulse length).

Open Access This article is distributed under the terms of the Creative Commons Attribution License which permits any use, distribution, and reproduction in any medium, provided the original author(s) and the source are credited.

\section{References}

1. J.-P. Besson, S. Schilt, L. Thevenaz, Proc. SPIE 5855, 415 (2005)

2. A. Miklós, S.-C. Pei, A.H. Kung, Appl. Opt. 45, 2529 (2006)

3. A. Miklos, P. Hess, Rev. Sci. Instrum. 72, 1937 (2001)

4. S. Schafer, A. Miklos, P. Hess, Appl. Opt. 36, 3202 (1997)

5. T. Borowski, T. Starecki, Eur. Phys. J. Spec. Top. 153, 439 (2008)

6. R. Kastle, M.W. Sigrist, Appl. Phys. B 63, 389 (1996)

7. J. Pelzl, K. Klein, O. Nordhaus, Appl. Opt. 21, 94 (1982)

8. G.Z. Angeli, Z. Bozoki, A. Miklos, A. Lorincz, A. Thony, M.W. Sigrist, Rev. Sci. Instrum. 62, 810 (1991)

9. R. Bartlome, M. Kaucikas, M.W. Sigrist, Appl. Phys. B 96, 561 (2009)

10. M. Suchenek, T. Starecki, Int. J. Thermophys. 32, 893 (2011)

11. M. Szakall, A. Varga, A. Pogany, Z. Bozoki, G. Szabo, Appl. Phys. B 94, 691 (2009) 